\title{
Clinical Features of Head and Neck Solitary Extramedullary Plasmacytoma in Taiwan
}

\author{
CHUN-YU LAI ${ }^{1}$, HUA-HSIN HSIEH ${ }^{1}$, HOU-KUANG CHEN ${ }^{1}$, CHE-YI CHAO ${ }^{2}$, CHUN-HUNG HUA ${ }^{1}$, \\ CHIH-JAAN TAI ${ }^{1}$, DA-TIAN BAU ${ }^{3,4}$, MING-HSUI TSAI ${ }^{1,3}$ and LIANG-CHUN SHIH ${ }^{1,3}$ \\ ${ }^{1}$ Department of Otorhinolaryngology, China Medical University Hospital, Taichung, Taiwan, R.O.C; \\ ${ }^{2}$ Department of Food Nutrition and Health Biotechnology, Asia University, Taichung, Taiwan, R.O.C.; \\ ${ }^{3}$ Graduate Institute of Biomedical Sciences, China Medical University, Taichung, Taiwan, R.O.C; \\ ${ }^{4}$ Department of Bioinformatics and Medical Engineering, Asia University, Taichung, Taiwan, R.O.C.
}

\begin{abstract}
Background/Aim: Solitary extramedullary plasmacytoma (SEP) is a rare, malignant plasma-cell tumor, which mainly occurs in the head and neck regions. Globally the disease has been rarely happening up to 2019, with only about ten papers focused on SEP cases reported in English. Thus, a literature collectively reviewing the characteristics of the patients would be valuable. Patients and Methods: We enrolled 10 SEP patients, and recorded their primary sites and the treatment modality, and analyzed their survival rates and outcomes. We also reviewed previous studies and compared their findings with ours. Results: No gender or age disparity has been observed, and younger patients had a better local control with RT compared to surgery among our patients. Conclusion: Further investigations with more patients and long-time follow-up may provide more information for treatment determination and the recurrence and progression from SEP to MM.
\end{abstract}

Plasmacytoma is a form of cancer derived from neoplastic monoclonal plasma cells, and it can be classified into two groups depending on its location: i) solitary extramedullary plasmacytoma (SEP) and ii) solitary plasmacytoma of bone (SPB) (1). Plasmacytoma accounts for approximately $4 \%$ of plasma cell malignancies. Multiple myeloma (MM) is the systemic form of the disease and the most common type of

This article is freely accessible online.

Correspondence to: Liang-Chun Shih, Ming-Hsui Tsai and Da-Tian Bau, Department of Otorhinolaryngology, China Medical University Hospital, 2 Yuh-Der Road, Taichung, Taiwan, R.O.C. Tel: +886 422052121 Ext 4436, e-mail: entdrshih7111@gmail.com (L-C Shih)

Key Words: Solitary extramedullary plasmacytoma, head and neck, Taiwan. plasma cell tumors, presenting with multiple lesion sites (2$6)$. The differential diagnosis for both SEP and SPB from $\mathrm{MM}$ is a lack of CRAB features (increased calcium, renal insufficiency, anemia, or multiple bone lesions) (2-6). Approximately $80 \%$ of SEP cases arise in the submucosa over the head and neck region, and the most common sites are the sinonasal/nasopharyngeal area, producing epistaxis, nasal discharge, or nasal obstruction. Less common sites, including the thyroid, breast, gastrointestinal tract, liver, lung, skin, CNS, and urinary bladder, are also reported (4, 7). The diagnostic criteria for SEP include: i) a tissue biopsy indicating monoclonal plasma cell histology, ii) bone marrow plasma cell infiltration less than $5 \%$ of all nucleated cells, iii) absence of osteolytic bone lesions or other tissue involvement without proof of myeloma, iv) hypercalcemia or renal failure, and v) a low serum $\mathrm{M}$ protein concentration in plasma or urine, if present (1). There are also typical pictures under microscopy including round and eccentric nuclei, with dense chromatin clumps surrounding by nuclear membrane. Due to its monoclonal nature, the neoplasm will produce either kappa or lambda light chain ( $\mathrm{k}$-chain, $\lambda$ chain), which can be used to rule out other diagnoses such as undifferentiated carcinoma $(2,4)$. The median age at diagnosis of SEP is between 55 and 60 years, and approximately two thirds of patients are male (6-9). Current series about SEP are mainly reports from the West.

As a matter of fact, there is a great difference among ethics in the initial presentation and disease progression, however the importance of genetic variability, diet and lifestyle, as well as environmental factors for the development of SEP is seldom revealed. In this study, we aimed to examine SEP patients in a medical center in Taiwan and assess their clinical image, treatment outcomes, survival rates, and immunohistochemical characteristics. Most valuable, we have reviewed several SEP studies in literature and compared and discussed their results with ours. 


\section{Patients and Methods}

The clinicians at the department of otorhinolaryngology, who are all well-trained surgeons, have reviewed the database from SEP diagnosed patients at the cancer center of the China Medical University Hospital, from 2010/01/01 to 2017/12/31. Each record of patients has been reviewed and double-checked by at least two clinicians.

The diagnostic criteria for SEP should be fulfilled to exclude MM $(9,10)$ and are the following: i) pathological proof of monoclonal plasma cells at a single extramedullary site, ii) negative clonal plasma cell involvement in bone marrow aspiration, iii) no anemia, hypercalcemia, or renal impairment attributed to plasma cell dyscrasias, iv) a low level of monoclonal immunoglobulin in the serum or urine, and v) no osteolytic lesion following a skeletal survey of the spine and pelvis. The patients' charts were reviewed for the following indexes: i) basic characteristics, ii) initial presentation, iii) lesion location, iv) treatment modalities, v) followup time and vi) survival outcome.

The immunohistochemically translational expression levels in the tissues were examined. We checked staining with $\mathrm{K}$-light chain, $\lambda$ light chain, cytokeratin, CD3, CD20, CD38 (Leica Microsystems Inc., Buffalo Grove, IL, USA), CD56 (Zytomed Systems GmbH, Berlin, Germany) and CD138 (Zeta Corporation, CA, USA).

\section{Results}

As shown in Table I, ten patients were diagnosed with SEP in this study, five of which were men $(50 \%)$. The mean age was 54.7 years (range $=37-73$ years). Seven cases $(70 \%)$ were located over the head and neck region and three (30\%) were not located in the head and neck area (inguinal, lung, and stomach) (Table II). Out of the seven cases two (20\%) were in the nasopharynx region, one $(10 \%)$ in the tonsil, two $(20 \%)$ in the supraglottic area, one $(10 \%)$ over the scalp and one $(10 \%)$ over the neck. There were three cases. For the seven cases of SEP over the head and neck region (Table III), our treatment modality was mainly radiation therapy (cases 2, 4, 6 , and 7), while cases 1,3 , and 5 received only surgery. All seven cases had been verified by immunochemistry for immunoglobulin light-chain production, and 5 of 7 cases (71\%) were $\mathrm{K}$-chain predominant, while the other 2 were $\lambda$ chain predominant. Our longest follow-up time was 94 months, and the shortest one was 12 months. During the follow-up, two cases ( 3 and 5) progressed to MM: case 3 following initial surgery alone and case 5 following initial radiation therapy. Three cases $(2,6$, and 7$)$ that initially received radiation therapy had no evidence of disease. Case 1 was initially treated with surgical excision; however, a second lesion was noted in the lung after 7 years of followup, and this patient is now under chemotherapy. Case 4 received surgery at first and then was lost to follow-up.

\section{Discussion}

Plasmacytoma is a rare disease with an incidence of 0.34 per 100,000 people/year, while MM is approximately 16 times
Table I. Characteristics of the investigated SEP patients.

\begin{tabular}{lc}
\hline Character & $\mathrm{N}(\%)$ \\
\hline Gender & \\
Male & $5(50 \%)$ \\
Female & $5(50 \%)$ \\
Age & \\
$<50$ & $4(40 \%)$ \\
$0-60$ & $2(20 \%)$ \\
$\geq 60$ & $4(40 \%)$ \\
Mean \pm SD & $54.7 \pm 11.9$ \\
\hline
\end{tabular}

SD: Standard deviation.

Table II. Primary sites of the investigated SEP patients.

\begin{tabular}{ll}
\hline Site & $\mathrm{n}(\%)$ \\
\hline Nasopharynx & $2(20 \%)$ \\
Tonsil & $1(10 \%)$ \\
Supraglottic & $2(20 \%)$ \\
Neck & $1(10 \%)$ \\
Scalp & $1(10 \%)$ \\
Inguinal & $1(10 \%)$ \\
Lung & $1(10 \%)$ \\
Stomach & $1(10 \%)$ \\
\hline
\end{tabular}

more frequent, with an incidence of 4 per 100,000 people/year. Among the plasmacytomas, SEB is approximately $40 \%$ more frequent compared to SEP, bearing different presentation features, for example SEB mostly occurs in the axial skeleton, such as the spinal column and skull (5). Nevertheless, SEP is mainly localized in the nasopharyngeal area. The etiology of SEP remains unknown; however, it may be related to chronic stimulation of inhaled irritants or viral infection to the mucosa of the aerodigestive tract $(>80 \%)(4,8-10)$. We have reviewed several studies and compared their results to ours.

Several studies have shown consistent results for the mean age at diagnosis of SEP (55 to 60 years) and gender disparity (male predominance) (2-6). The same distributive characteristics were mentioned in Taiwan 2 decades earlier (11). In our study, $50 \%$ of patients were male, and the mean age at diagnosis was 54.7 years. Though our data showed no gender disparity, possibly due to the small number of patients sampled, the mean age was similar to what has been reported in Western studies. Moreover, according to the literature, age has a more significant role in determining prognostic factors compared to gender (12). The favorable prognostic factor for multiple-myeloma-free survival in SEP patients is a younger age, and treatment outcomes in younger 

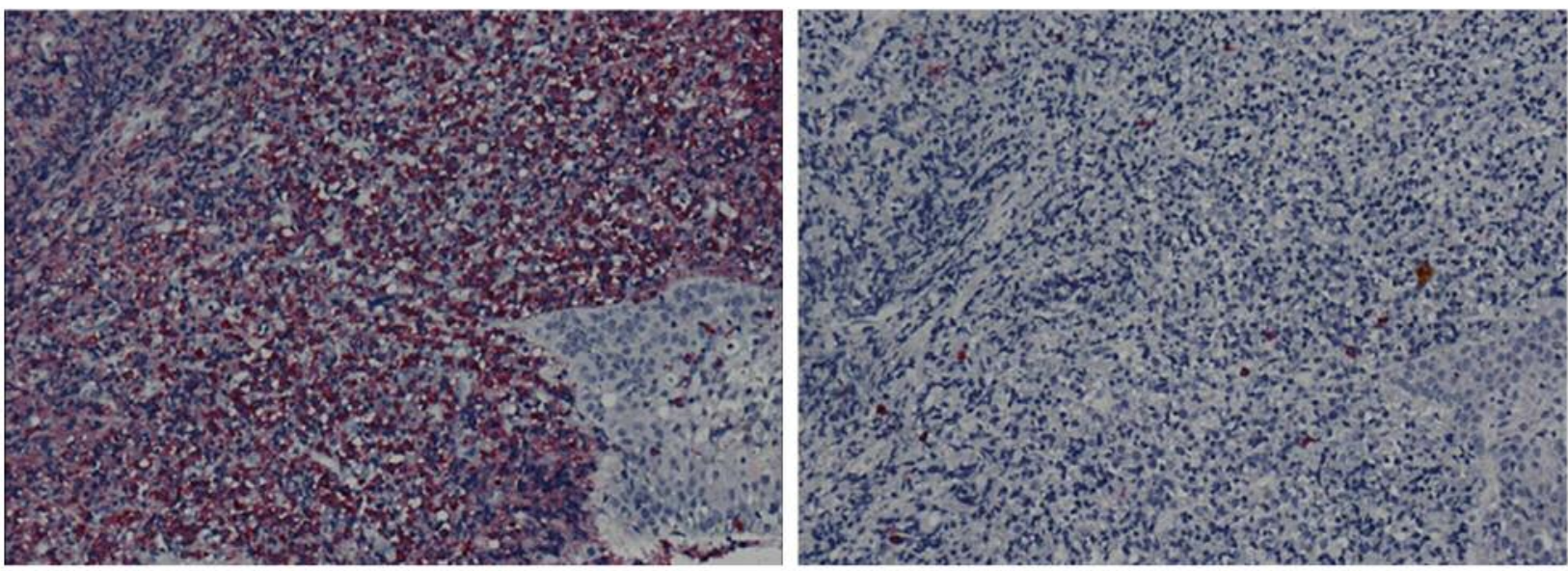

Figure 1. Figure 1. Immunostaining of tissue samples from left: typical $k$-chain predominant, and right: $\lambda$-chain predominant types of SEP patients. Scale bars:200x.

Table III. Summary of the basic and clinical features for the seven head and neck SEP patients.

\begin{tabular}{|c|c|c|c|c|c|}
\hline Case & Age/Gender & Site & $\mathrm{k} / \lambda$ restriction & Follow-up time & Therapy, prognostic status \\
\hline 1 & $63 / \mathrm{F}$ & Right false cord & $\mathrm{K}$ & $7 \mathrm{y} 10 \mathrm{~m}$ & OP, lung involved in $2017 \rightarrow \mathrm{CT}$ \\
\hline 2 & $37 / \mathrm{M}$ & Nasopharynx & $\mathrm{K}$ & 6 y $8 \mathrm{~m}$ & RT, NED \\
\hline 3 & $47 / \mathrm{F}$ & Right lower neck & $\lambda$ & 5 y $5 \mathrm{~m}$ & $\mathrm{OP}$, progressed to $\mathrm{MM}$ \\
\hline 4 & $56 / \mathrm{M}$ & Left scalp & $\lambda$ & $2 \mathrm{y} 0 \mathrm{~m}$ & OP, lost follow up \\
\hline 5 & $60 / \mathrm{M}$ & Right tonsil & $\mathrm{K}$ & $4 \mathrm{y} 0 \mathrm{~m}$ & $\mathrm{RT}$, progressed to $\mathrm{MM}$ \\
\hline 6 & $45 / \mathrm{F}$ & Left nasopharynx & $\mathrm{K}$ & 3 y $2 \mathrm{~m}$ & RT, NED \\
\hline 7 & $74 / \mathrm{M}$ & Right supraglottic & $\mathrm{K}$ & $1 \mathrm{y} 0 \mathrm{~m}$ & RT, NED \\
\hline
\end{tabular}

M: Male; F: female; y: year; m: month; OP: operation; CT: chemotherapy; RT: radiotherapy; NED: no evidence of disease; MM: multiple myeloma.

patients seem to follow a similar trend. Gender, however, does not appear to have a significant effect on the outcome of these patients (11).

The head and neck region, undoubtedly, is known to be the most common lesion site for SEP, including the nasal cavity, sinuses, nasopharynx, and larynx $(4,7)$. In our study, the primary sites differed but mainly occurred in the head and neck region $(7 / 10,70 \%)$, including the nasopharynx, tonsil, supraglottic area, neck, and scalp. Interestingly, as already mentioned, lesions may occur in virtually any organ. Three cases $(30 \%)$ in our study presented with primary sites outside the head and neck region, including the lungs, inguinal region, and stomach.

Tissue proof of monoclonal plasma cell histology is one of the diagnostic criteria of SEP. Predominance of monoclonal IgA has been reported as an indicator (13). A biopsy-based diagnosis is more easily accomplished by testing for CD38 positivity for phenotypic studies and monoclonal light chain expression of malignant plasma cells from the solitary lesion (7). Five out of seven cases (71\%) in our data-base were $\mathrm{K}$-chain predominant, and the other two were $\lambda$-chain predominant $(29 \%)$ (Figure 1), compatible with the results of a literature review, showing $65 \%$ prevalence of $\mathrm{K}$-chain predominant cases $(2,4)$.

Our longest follow-up time was 94 months, and the shortest one was 12 months, with the average follow-up time being 51 months (4.25 years). Since the natural course of plasmacytoma progression to multiple-myeloma (MM) requires years, longterm follow-up provides valuable information for the prognosis as well as for further medical investigation, such as progression rate, treatment outcomes, and recurrence rate.

SEP is highly radiosensitive. Radiation therapy is currently the first line of treatment, with a local control rate reaching up to $90 \%$. It has been reported that the 10 -year disease-free and overall-survival rates range from $50 \%$ to $80 \%$, and fewer than $10 \%$ of patients have local recurrence (2-10). However, about $30 \%-50 \%$ of patients can progress to $\mathrm{MM}$ in a mean period ranging from 1.5 to 2.5 years (7). Statistical results vary from study to study (2-10), but the overall results appear to be compatible. 
Since $30 \%-50 \%$ of the patients progress to MM, it is critical to determine statistically significant risk factors and define practicable dose guidelines for radiation therapy. Unfortunately, there is no consensus regarding the risk factors that cause plasmacytoma to progress to MM. Regarding radiation dosing, the recommended curative dose is 40-50 Gy over a 4-week period, but strict RT dosing guidelines are still difficult to confirm due to the lack of prospective studies, and the relation between RT dose and progression-free survival needs further explanation (12). Two of our cases progressed to MM (2/7, 29\%), with one receiving surgery and the other receiving radiation therapy only. Regarding our local control rate with radiation therapy, three cases $(3 / 7,43 \%)$ demonstrated no local recurrence.

The role of surgery in this disease remains uncertain, and for adjuvant chemotherapy, it is much more debatable. Still, many series have mentioned the role of primary surgical resection in patients with lesions in areas other than the head and neck $(2,4,5,12)$; moreover, some series have claimed the benefits of combining RT with surgery $(14,15)$. If a complete surgical resection is performed at diagnosis, the role of adjuvant RT is unclear (9). Small lesions may be cured with surgery alone, and no adjuvant RT is indicated unless there is a residual tumor $(2,4,5,12)$.

Regarding the outcome of plasmacytoma, SEP is well known to be better compared to SBP. However, the main concern is with regard to the multiple-myeloma-free survival (MMFS) and the local recurrence rate of SEP itself. Several studies have endeavored to clarify the prognostic factors of SEP but could not arrive at a consensus. Kilciksiz et al. (1) have compared the outcomes of RT to those of surgery alone in 80 patients with the following factors: i) progression-free survival (PFS) and ii) MMFS. The combination of radiotherapy and surgery had a positive effect on PFS for the overall plasmacytoma group and SPB subgroup but notably not for the SEP group. For SEP patients, the favorable factor was younger age for MMFS. RT at >50 Gy and RT combined with surgery may be favorable prognostic factors in PFS (12).

Up to the end of our study, none of our patients died, and the overall survival rate was $86 \%$ (6/7, 1 was lost to follow-up). Due to the insufficient number of patients, we could not confirm longer MMFS for the younger age group, but we observed a probable tendency in our result: among the three cases with no recurrence, two of them were $<50$ years old $(66.7 \%)$, and a reasonable explanation for the third one with negative recurrence could be that the follow-up time (1 year) was below the mean period of progression to MM (1.5-2.5 years).

Furthermore, of all four patients who received RT alone and had been followed up for at least 3 years, the younger patients presented a better MMFS compared to the older one. Moreover, the reverse was also true: three cases who were all $<50$ years old and had been followed up for at least 3 years probably had a better local control with RT alone compared to surgery.
Our institute has faced a similar dilemma to other databases concerning plasmacytoma, which is an insufficient number of cases. Nevertheless, even with a limited database, we endeavored to compare our results to those present in the literature, and most of them produced comparable results. Only few studies in Taiwan have investigated plasmacytoma and have been followed up during a long time, so our contribution to this is valuable. Longer follow-up of these patients is necessary due to the natural course of plasmacytoma and MM. We hope to conclude more valuable information about the treatment and prognostic factors in the future via performing a longer follow-up by ourselves.

SEP predominantly occurs in the submucosa of the head and neck region, with RT being the first line of treatment for SEP. Differential diagnosis between SEP and MM is important because SEP has a high risk of recurrence and progression to MM. Thus, regular radiological and electrophoresis assessment during follow-up are suggested for patients with SEP. Further studies enrolling more patients from multiple centers and over a longer time course may provide more information about the recurrence and progression from SEP to MM.

\section{Conflicts of Interest}

All Authors declare that: i) no support, financial or otherwise, has been received from any organization that may have an interest in the submitted work, and ii) there are no other relationships or activities that could appear to have influenced the submitted work.

\section{Authors' Contributions}

Research design: Lai CY, Tsai MH and Shih LC; Patient and questionnaire summarize: Hsieh $\mathrm{HH}$, Chen $\mathrm{HK}$ and $\mathrm{Hua} \mathrm{CH}$; Experiment performance: Hsieh $\mathrm{HH}$ and Chen HK; Statistical analysis: Chao CY and Tai CJ; Manuscript writing: Shih LC and Bau DT; Reviewing and revising: Tsai MH, Shih LC and Bau DT.

\section{Acknowledgements}

This work was conducted in Department of Otorhinolaryngology in China Medical University Hospital, and partially supported by the grant from China Medical University Hospital (CMU108-ASIA-01) to Dr. Shih.

\section{References}

1 Kilciksiz S, Karakoyun-Celik O, Agaoglu FY and Haydaroglu A: A review for solitary plasmacytoma of bone and extramedullary plasmacytoma. Sci World J 2012: 895765, 2012. PMID: 22654647. DOI: 10.1100/2012/895765

2 Susnerwala SS, Shanks JH, Banerjee SS, Scarffe JH, Farrington WT and Slevin NJ: Extramedullary plasmacytoma of the head and neck region: clinicopathological correlation in 25 cases. $\mathrm{Br}$ J Cancer 75: 921-927, 1997. PMID: 9062417. DOI: 10.1038/ bjc. 1997.162 
3 Shih LY, Dunn P, Leung WM, Chen WJ and Wang PN: Localised plasmacytomas in Taiwan: comparison between extramedullary plasmacytoma and solitary plasmacytoma of bone. Br J Cancer 71: 128-133, 1995. PMID: 7819027. DOI: 10.1038/bjc. 1995.26

4 Miller FR, Lavertu P, Wanamaker JR, Bonafede J and Wood BG: Plasmacytomas of the head and neck. Otolaryngol Head Neck Surg 119: 614-618, 1998. PMID: 9852535. DOI: 10.1016/ S0194-5998(98)70021-X

5 Pinto JA, Sonego TB, Artico MS, Leal Cde F and Bellotto S: Extramedullary plasmacytoma of the larynx. Int Arch Otorhinolaryngol 16: 410-413, 2012. PMID: 25991967. DOI: 10.7162/S1809-97772012000300019

6 Dores GM, Landgren O, McGlynn KA, Curtis RE, Linet MS and Devesa SS: Plasmacytoma of bone, extramedullary plasmacytoma, and multiple myeloma: incidence and survival in the United States, 1992-2004. Br J Haematol 144: 86-94, 2009. PMID: 19016727. DOI: 10.1111/j.1365-2141.2008.07421.x

7 Weber DM: Solitary bone and extramedullary plasmacytoma. Hematology Am Soc Hematol Educ Program 2005(1): 373-376, 2005. PMID: 16304406. DOI: 10.1182/asheducation-2005.1.373

8 Al Saey H, Larem A, Al Sulaiti M, Shanmugam G, Mujeeb I and Bhat V: Solitary Extramedullary plasmacytoma of the Larynx presenting with stridor as a primary manifestation- A case report Qatar Medical Journal 21: 39-43, 2011. DOI: 10.5339/qmj.2011. 2.16

9 Krebs S, Ganly I, Ghossein R, Yang J, Yahalom J and Schoder $\mathrm{H}$ : Solitary extramedullary plasmacytoma of the cricoid cartilage-case report. Front Oncol 7: 284, 2017. PMID: 29230383. DOI: $10.3389 /$ fonc.2017.00284

10 Merza H and Sarkar R: Solitary extraosseous plasmacytoma. Clin Case Rep 4: 851-854, 2016. PMID: 27648261. DOI: $10.1002 /$ ccr3.609
11 Kilciksiz S, Celik OK, Pak Y, Demiral AN, Pehlivan M, Orhan O, Tokatli F, Agaoglu F, Zincircioglu B, Atasoy BM, Ozseker N, Yersal O, Niang U, Haydaroglu A and Turkish Oncology GroupSarcoma Working P: Clinical and prognostic features of plasmacytomas: a multicenter study of Turkish Oncology GroupSarcoma Working Party. Am J Hematol 83: 702-707, 2008. PMID: 18543343. DOI: 10.1002/ajh.21211

12 International Myeloma Working Group: Criteria for the classification of monoclonal gammopathies, multiple myeloma and related disorders: a report of the International Myeloma Working Group. Br J Haematol 121: 749-757, 2003. PMID: 12780789

13 Shih L, Chen J, Tsou Y, Tseng G, Tsai M, Chang H, Huang K, Chen D and Chen C: Multiple extramedullary plasmacytoma involving the vallecula: A case report with 3 -year follow-up. Head Neck Oncol 6: 6, 2014.

$14 \mathrm{Hu} \mathrm{K}$ and Yahalom $\mathrm{J}$ : Radiotherapy in the management of plasma cell tumors. Oncology (Williston Park) 14: 101-108, 111; discussion 111-102, 115, 2000. PMID: 10680152.

15 Galieni P, Cavo M, Pulsoni A, Avvisati G, Bigazzi C, Neri S, Caliceti U, Benni M, Ronconi S and Lauria F: Clinical outcome of extramedullary plasmacytoma. Haematologica 85: 47-51, 2000. PMID: 10629591.
Received September 21, 2019

Revised October 5, 2019

Accepted October 10, 2019 